\title{
Survey of the ULF wave Poynting vector near the Earth's magnetic equatorial plane
}

\author{
M. D. Hartinger, ${ }^{1}$ M. B. Moldwin, ${ }^{1}$ K. Takahashi, ${ }^{2}$ J. W. Bonnell, ${ }^{3}$ and V. Angelopoulos ${ }^{4}$ \\ Received 12 August 2013; revised 16 September 2013; accepted 23 September 2013; published 7 October 2013.
}

[1] Ultralow frequency (ULF) waves transfer energy in the Earth's magnetosphere through a variety of mechanisms that impact the Earth's ionosphere, radiation belts, and other plasma populations. Measurements of the electromagnetic portion of the energy transfer rate are an important source of information for assessing the importance of ULF waves relative to other energy transfer mechanisms as well as a diagnostic for studying the behavior of ULF waves. Using Time History of Events and Macroscale Interactions during Substorms satellite data, we examine the time-averaged electromagnetic energy transfer rate, or Poynting vector, as a function of frequency and region of the magnetosphere; for this study, we focus on the direction and rate of energy transfer relative to the background magnetic field, comparing perpendicular and parallel transfer rates. This study extends earlier studies of the ULF wave Poynting vector that focused on narrower frequency ranges or specific regions of the magnetosphere; here we consider the $3-50 \mathrm{mHz}$ frequency range, all local time sectors, radial distances from 3 to $13 \mathrm{Re}$, and magnetic latitudes close to the equatorial plane. We measure time-averaged Poynting vectors that range from $10^{-11}$ to $10^{-5} \mathrm{~W} / \mathrm{m}^{2}$, with larger Poynting vector magnitudes occurring at larger radial distances and smaller frequencies. In every spatial region and frequency we examined, we found a large degree of scatter in both the Poynting vector magnitude and direction. The Poynting vector tends to be anisotropic at all frequencies, with more energy transferred along rather than across the background magnetic field. This preference for parallel energy transfer near the magnetic equator suggests that Joule dissipation in the ionosphere and the acceleration of auroral electrons are the largest sinks of ULF wave energy in the magnetosphere.

Citation: Hartinger, M. D., M. B. Moldwin, K. Takahashi, J. W. Bonnell, and V. Angelopoulos (2013), Survey of the ULF wave Poynting vector near the Earth's magnetic equatorial plane, J. Geophys. Res. Space Physics, 118, 6212-6227, doi:10.1002/jgra.50591.

\section{Introduction}

[2] Ultralow frequency (ULF) waves are an important mechanism for energy transfer in the Earth's magnetosphere. They have frequencies less than $5 \mathrm{~Hz}$ and include a wide range of wave phenomena [Jacobs et al., 1964]. For example, depending on frequency and location in the Earth's magnetosphere, some ULF waves may be described well

Additional supporting information may be found in the online version of this article.

${ }^{1}$ Department of Atmospheric, Oceanic, and Space Sciences, University of Michigan, Ann Arbor, Michigan, USA.

${ }^{2}$ Applied Physics Laboratory, Johns Hopkins University, Laurel, Maryland, USA.

${ }^{3}$ Space Science Laboratory, University of California, Berkeley, California, USA

${ }^{4}$ Department of Earth and Space Sciences, University of California, Los Angeles, California, USA.

Corresponding author: M. D. Hartinger, Space Research Building, University of Michigan, 2455 Hayward St., Ann Arbor, MI 48109-2143, USA. (mdhartin@umich.edu)

(C2013. American Geophysical Union. All Rights Reserved 2169-9380/13/10.1002/jgra.50591 by magnetohydrodynamics (MHD) whereas others require a two-fluid or fully kinetic description. In this work, we shall consider waves in the $3-50 \mathrm{mHz}$ frequency range $(20-333 \mathrm{~s})$, which are usually well described by ideal MHD.

[3] Three widely studied examples that illustrate the importance of ULF waves for energy transfer are Joule dissipation in the ionosphere/auroral acceleration, wave-particle interactions with outer radiation belt electrons, and field line resonances (FLR). Alfvén waves transfer energy along the field line and toward the ionosphere; this energy is lost to Joule dissipation in the ionosphere and the field-aligned acceleration of auroral electrons [e.g., Rae et al., 2007; Lysak and Song, 2003; Rae et al., 2012]. In some cases, the energy lost to the ionosphere is comparable to the energy released in a substorm [Greenwald and Walker, 1980; Rae et al., 2007]. Radial diffusion and drift resonance via ULF waves are important mechanisms that can affect the population of relativistic electrons in the outer radiation belt [e.g., Elkington et al., 2003]. FLR is a resonant coupling between the shear Alfvén and compressional MHD wave modes; it provides an explanation for the buildup of wave energy at discrete frequencies and locations in the Earth's magnetosphere [Tamao, 1965]. 
[4] A number of statistical studies examined electromagnetic energy transfer rates, or the Poynting vector $(\vec{S})$, associated with ULF waves in the Earth's magnetosphere. Keiling et al. [2002, 2003] found that parallel (to background magnetic field) Poynting vectors associated with ULF waves (6-180 s) provided energy for the aurora. Loto'aniu et al. [2005] examined parallel Poynting vectors associated with electromagnetic ion cyclotron (EMIC) waves, finding that the direction of energy transfer rapidly changed for some events. Chi and Russell [1998] examined Poynting vectors associated with Pc3-Pc4 (10-150 s-overlaps with Pi2 band, 45-150 s) waves, documenting the "phase-skipping" phenomenon, or the preference of waves to rapidly change propagation direction. Junginger et al. [1985] studied Poynting vectors associated with Pc5 (150-600 s) waves at geosynchronous orbit, finding values of $10^{-10}$ to $10^{-5} \mathrm{~W} / \mathrm{m}^{2}$ (note that these values, when mapped along a field line to the ionosphere, are on the order of several $\mathrm{mW} / \mathrm{m}^{2}$ ) [e.g., Rae et al., 2007; Hartinger et al., 2011]; they also noted the seasonal effect of the ionospheric conductivity on parallel energy transfer rates. Sakurai et al. [2001] examined $\vec{S}$ for Pc5 waves in the outer magnetosphere, finding values comparable to Junginger et al. [1985] and estimating total energy transferred from the magnetopause to the inner magnetosphere over $1 \mathrm{~h}$ of continuous Pc5 wave activity at $10^{10}$ to $10^{13} \mathrm{~J}$.

[5] Comparisons between ULF wave magnetic power spectral densities at different frequencies and in different regions of the magnetosphere reveal marked differences in wave properties and intensities [Anderson et al., 1990; Takahashi and Anderson, 1992]. Such comparisons have not been made using the ULF wave Poynting vector, since previous studies of electromagnetic energy transfer rates have focused on narrow frequency ranges and only certain regions of the magnetosphere. There are several open questions that could be answered through a systematic study of ULF wave Poynting vectors at different frequencies and in different regions of the magnetosphere: How do energy transfer rates at low frequencies compare to higher frequencies? What is the direction of energy transfer in different regions? Is there a preference for field-aligned versus perpendicular energy transport in different regions? The answers to these questions can be used to better predict the behavior of ULF waves and assess their role in driving space weather phenomena.

[6] Our purpose in this study is to quantify the direction and magnitude of the ULF wave time-averaged Poynting vector at different frequencies and locations in the Earth's magnetosphere. In section 2, we describe the instruments and data sources used for this study. In section 3 , we describe the techniques we used for data calibration, signal processing, and data reduction. In section 4, we present observations of the Poynting vector at different frequencies and in different regions and discuss their implications. In section 5, we summarize our results.

\section{Instrumentation}

[7] We use data from the five probe Time History of Events and Macroscale Interactions during Substorms (THEMIS) mission to study the ULF wave Poynting vector [Sibeck and Angelopoulos, 2008]. For the probes used in this study (THEMIS A (THA), D (THD), and E (THE)), the perigee was $1.5 \mathrm{Re}$, the apogee varied from 10 to $13 \mathrm{Re}$, and the inclination varied from $5^{\circ}$ to $12^{\circ}$ [Frey et al., 2008]. Each spinning probe ( $3 \mathrm{~s}$ spin period) is equipped with a fluxgate magnetometer (FGM) [Auster et al., 2008], an electric field instrument (EFI) [Bonnell et al., 2008], an ion and electron electrostatic analyzer (ESA) [McFadden et al., 2008], and ion and electron solid state telescopes (SST) [e.g., Turner et al., 2012]. ESA measures three-dimensional particle distributions and moments (electrons: $5 \mathrm{eV}$ to $30 \mathrm{keV}$, ions: $5 \mathrm{eV}$ to $25 \mathrm{keV}$ ) once per spin. SST measures the three-dimensional particle distributions and moments once per spin and is sensitive to energies above $25 \mathrm{keV}$. Finally, we obtained hourly geomagnetic activity indices from the Space Physics Data Facility (a project of NASA's Goddard Space Flight Center) OMNIWeb interface at http://omniweb. gsfc.nasa.gov.

\section{Methodology}

\subsection{Data Collection and Calibration}

[8] We obtain EFI, FGM, ESA, and SST data from the THEMIS website (http://themis.ssl.berkeley.edu/index. shtml) and apply the latest calibrations and corrections using the software package distributed by the THEMIS science team (version 07 January 2013). We analyzed data from the period 01 February 2008 to 01 December 2012 - computing $\vec{S}$ requires electric field data, and these data were not available routinely before February 2008 . We chose to use data from three THEMIS probes that spent the most time in the magnetosphere during this period: THA, THD, and THE. For wave analysis, we only use data when these probes were in fast survey mode. During these periods, ground calibrations can be applied, higher resolution data are available, and the data are generally higher quality. Furthermore, several diagnostics can be used during these periods to identify intervals with contaminated electric field data [Hartinger et al., 2012, 2013].

\subsection{Signal Processing}

[9] Much of the preliminary signal processing is similar to the procedure used by Hartinger et al. [2013]. Most of the THEMIS data quantities (excluding ephemeris) have time resolution of roughly $3 \mathrm{~s}$ (spacecraft spin period) or higher. We remove small gaps from the data $(<4 \mathrm{~s})$ and resample all data sets to the same time resolution-3 s-using nearest neighbor interpolation. We exclude all magnetosheath, low radial distance $(<3 \mathrm{Re}$ - wave quantities cannot be reliably computed in this region), and slow survey data intervals from our wave analysis. We define magnetosheath periods as intervals when the probe is at a radial distance greater than $8 \mathrm{Re}$ and the measured electron density is greater than 5 particles $/ \mathrm{cm}^{3}$ or the perpendicular flux (ESA) is greater than $2 \times 10^{7}$ particles $/ \mathrm{s} / \mathrm{cm}^{2}$. Of the remaining data, we further exclude intervals that are shorter than $1 \mathrm{~h}$; we require this length for detrending and Fourier analysis.

[10] We remove spikes from both the electric and magnetic field data using two techniques. Initially, we estimate the first derivative by calculating the difference between measurements made at subsequent $3 \mathrm{~s}$ time steps; we use the magnitude of the derivative as a filter to remove spikes, excluding periods with first derivatives greater than $10 \mathrm{mV} / \mathrm{m} / \mathrm{s}$ or $10 \mathrm{nT} / \mathrm{s}$. We further remove nongeophysical 
square wave type intervals from the magnetic field data (which happen only occasionally, last roughly $1 \mathrm{~min}$, and occur near a transition from fast to slow survey mode between 4.4 and $4.8 \mathrm{Re}$ radial distance) using comparisons with a model magnetic field.

[11] After spikes have been removed, we interpolate across gaps that are smaller than 12 s. Finally, we highpass filter (frequency $>2 \mathrm{mHz}$ ) the electric and magnetic field data and rotate these data into both GSM coordinates and a field-aligned (FA) coordinate system in which $z$ is along the background magnetic field, $y$ points eastward, and $x$ completes the right-hand orthogonal set (pointing radially outward at the equator); the background field for the FA coordinate transformation is obtained by low-pass filtering the magnetic field data (frequency $<2 \mathrm{mHz}$ ) - to maximize useable data and reduce filtering edge effects, we include both fast and slow survey data to compute the background field, since it is not used to calculate wave quantities.

[12] We remove the first and last 15 min of each fast survey interval to exclude portions of the signal that may be affected by the filtering process and data spikes occurring at the transition between fast and slow survey modes. We then compute the complex Fourier coefficients of the magnetic and electric field data using a 512 point, $25.6 \mathrm{~min}$ fast Fourier transform (FFT) window with no overlap between windows. A Hanning window is applied to the signal before performing the FFT; the Fourier coefficients are scaled to account for the signal attenuation associated with the Hanning window before they are used to obtain wave quantities (i.e., wave power spectral densities and Poynting vector magnitudes are not reduced or otherwise affected by the application of the Hanning window).

\subsection{Obtaining Wave Quantities}

[13] We compute two main wave quantities using the complex FFT coefficients: power spectral density (PSD) and the Poynting vector. The root-mean-square (RMS) amplitude can be obtained from the PSD's shown in this study using

$$
\text { Amplitude }=2 \sqrt{2 \Delta f \operatorname{PSD}(f)}
$$

where Amplitude is the RMS amplitude (units determined by units of PSD), $f$ is the frequency, $\Delta f$ is the width of the frequency bins in $\mathrm{Hz}(0.65 \mathrm{mHz})$, and $\operatorname{PSD}(f)$ is the power spectral density at each discrete frequency (units of $\mathrm{nT}^{2} / \mathrm{Hz}$ or $(\mathrm{mV} / \mathrm{m})^{2} / \mathrm{Hz}$ in this study). The frequency dependent Poynting vector is given by

$$
\vec{S}(f)=\frac{\Delta f}{\mu_{0}} \operatorname{Re}\left(\vec{E}(f) \times \vec{B}^{*}(f)\right)
$$

where $\vec{S}(f)$ is the frequency dependent Poynting vector in $\mathrm{W} / \mathrm{m}^{2}, \vec{B}(f)$ and $\vec{E}(f)$ are the complex Fourier coefficients for the magnetic and electric fields in $\mathrm{T}$ and $\mathrm{V} / \mathrm{m}$, respectively, * indicates the complex conjugate, $R e$ indicates the real part of the result, and $\mu_{0}$ is the permeability of free space. This technique for computing $\vec{S}(f)$ has been used recently for studies of EMIC waves and whistler mode chorus waves [Loto'aniu et al., 2005; Santolik et al., 2010; Li et al., 2013], and it is very similar to techniques previously used for studying Pc5 ULF waves [Junginger et al., 1985].

[14] Equation (2) is for the net energy transferred over the course of a given FFT window (it should not be regarded as an indicator of instantaneous energy transfer rate). Since the longest wave period considered in this study is roughly 4 times shorter than an FFT window ( $\sim 6$ min compared to $\sim 25.6 \mathrm{~min})$, this is a reliable indicator of the net energy transfer rate at a given frequency. Hereafter, when we refer to the Poynting vector $(\vec{S}(f))$, we are referring to the quantity computed using equation (2).

[15] Figure 1 shows a portion of one THE fast survey interval used in this study on 13 January 2010. From top to bottom, this figure shows the three FA components of the magnetic and electric perturbations, PSD for the magnetic field $y$ and electric field $x$ components, the component of $\vec{S}(f)$ directed along the field and toward the northern ionosphere, and the component of $\vec{S}(f)$ directed along the field and toward the southern ionosphere. To focus on the strongest wave activity, we show three point-averaged frequency bins and mask all $\vec{S}(f)$ data below $10^{-9} \mathrm{~W} / \mathrm{m}^{2}$. During this period, THE was close to the magnetic equator and on the inbound portion of its orbit; it observed harmonics consistent with damped, standing Alfvén waves and corresponding net (not instantaneous) electromagnetic energy transport primarily directed toward the northern ionosphere as the wave gradually lost energy. There was also transient wave activity which contributed to the energy transport along the background field; this, combined with the probe's location near the magnetic equator (near the null point for parallel energy transfer) [Allan, 1982], explains why there is some energy transport toward the southern ionosphere. Further from the equator, and in the absence of other wave modes, we expect all of the net energy transport for a damped, standing Alfvén wave to be directed either parallel or antiparallel to the background field. Our goal in aggregating $\vec{S}(f)$ data from many intervals such as these is to remove factors which may complicate the analysis of energy transport in individual events (e.g., superposition of multiple wave modes and wave reflection) and focus on statistical trends.

\subsection{Data Reduction}

[16] EFI is only sensitive to ULF perturbations in the spin plane of the spacecraft (the axial boom cannot routinely be used) [Bonnell et al., 2008]. We obtain the component along the spin axis using the $\vec{E} \cdot \vec{B}=0$ approximation; this approximation tends to have smaller random errors when the normal of the spin plane is at a large angle to the background magnetic field direction and when the component of the background magnetic field along the spacecraft spin axis is significantly larger than any magnetic field perturbations. Previous studies examining electric fields in the inner magnetosphere placed restrictions on the spin plane angle, typically requiring that it be larger than $20^{\circ}$ [e.g., Takahashi et al., 2003]. We require a more strict criteria, since our study includes data in regions with weaker background magnetic fields. We exclude EFI data when the spin axis component of the magnetic field is smaller than $30 \mathrm{nT}$ or when the ratio of the perpendicular (to spin axis) magnetic field components to the spin axis magnetic field is greater than 1 . This removes roughly half of the EFI data, mostly near the apogee of THEMIS (12-13 Re) and preferentially in the nightside magnetosphere. 


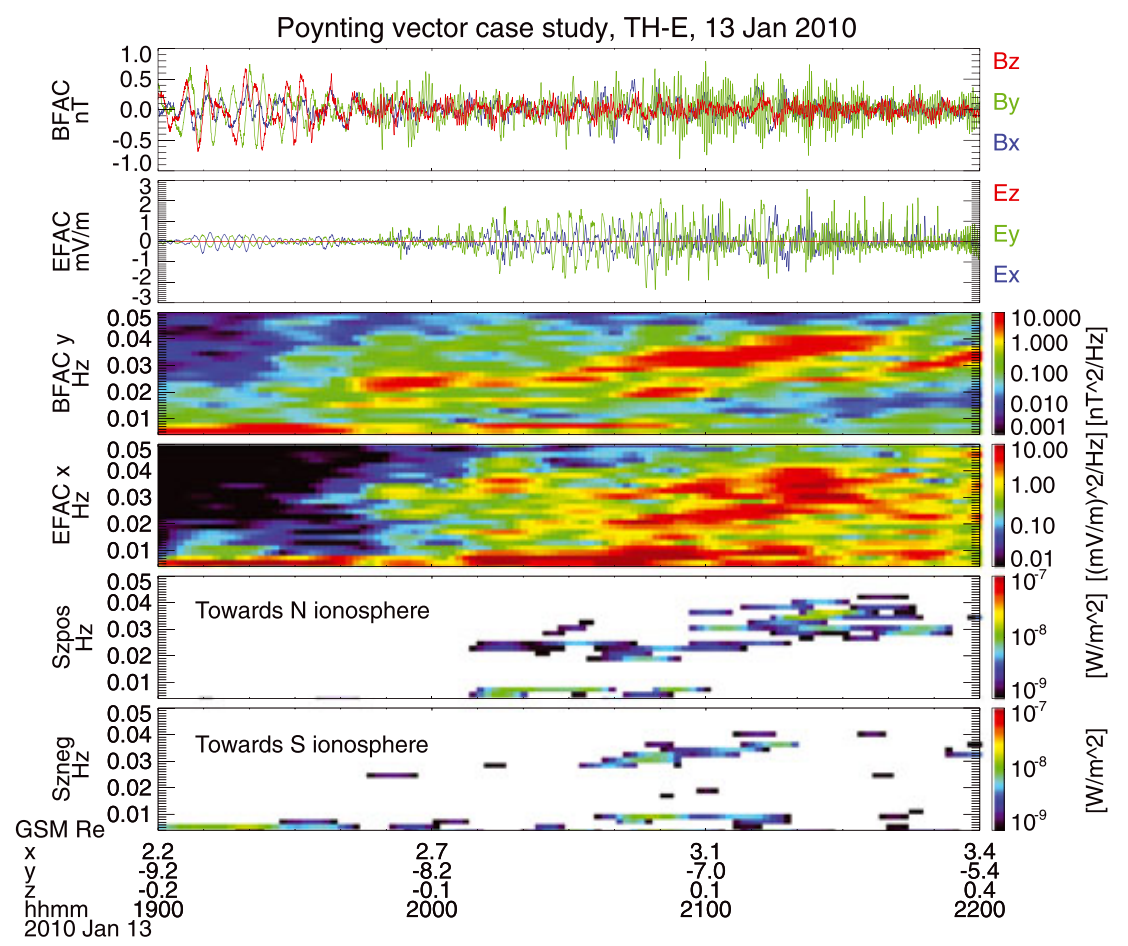

Figure 1. This figure shows an example of Poynting vector observations associated with standing Alfvén waves using the THE probe. (first and second panel) The perturbation magnetic and electric fields in FA coordinates in which $z$ is along the background magnetic field, $y$ points eastward, and $x$ is approximately radial. (third and fourth panel) The PSD for the magnetic field $y$ and electric field $x$ components, which are usually associated with toroidal mode standing Alfvén waves - there are multiple harmonics of standing waves present. (fifth and sixth panel) The parallel component of the Poynting vector in the direction toward the northern ionosphere and toward the southern ionosphere, respectively, with frequencies/times with small Poynting vectors masked to focus on strongest wave activity.

[17] EFI is also subject to contamination from electrostatic wakes and boom shadowing, affecting the ULF wave amplitude and phase measurement [Bonnell et al., 2008; Hartinger et al., 2012, 2013]. Following Hartinger et al. [2013], we used the phase difference between the spinfit electric fields measured by the short (40 m tip-to-tip) and long (50 m tip-to-tip) EFI booms (spin plane) as a diagnostic of electric field contamination. In the absence of contamination, these two booms should measure the same spinfit electric field perturbation (assuming the perturbation has a period much larger than $3 \mathrm{~s}$, the spin period) with a frequency dependent phase shift of $270^{*} f$ degrees; deviations from the expected phase shift indicate contamination, since the short boom is preferentially affected by contamination (it spends a longer time in the spacecraft shadow and is also more likely to sample the spacecraft wake). We found that the largest phase deviations occurred when the sun angle, or angle between the spacecraft spin plane and the ecliptic plane, was small $\left(-1.75^{\circ}<\right.$ sun angle $\left.<1.75^{\circ}\right)$; during these periods, the EFI booms spend the maximum amount of time in the shadow of the spacecraft and asymmetric illumination/photoemission-leading to spurious voltage differences measured in the spinning frame - is more likely to corrupt the spinfit fields. Large phase deviations also occurred in the presence of large fluctuations in the electron density (as inferred from spacecraft potential); these fluctuations generate electrostatic wakes that vary on ULF wave timescales-again leading to spurious, nongeophysical voltage differences in the spinning frame- that may corrupt the spinfit fields.

[18] We found that the most strongly contaminated intervals occurred when both of the above conditions were met: low sun angles and large density fluctuations. To reduce the effect of EFI contamination on our results while maximizing the amount of data used in our study, we exclude periods when the sun angle is low ( $\mid$ sun angle $\mid<1.75^{\circ}$ ). This does not remove all contaminated intervals; however, we found that remaining sources of contamination generated spurious electric fields with small amplitudes that were only noticeable when geophysical signals were very low. These contaminated intervals do not affect our results. As a further check on the data quality, we compared the ULF electric field perturbation with the electric field perturbation obtained using the ideal MHD assumption $E=-v \times B$, where $\mathrm{v}$ is the ion velocity moment measured by ESA and $\mathrm{B}$ is the background magnetic field, during periods when both measurements were expected to be reliable; they agreed well. Finally, we checked the data quality by comparing our results with previous studies that measured the electric field using different techniques, finding very close agreement (see section 4.1).

[19] We restrict to periods when there is a signal above the noise threshold of either instrument. We determined the noise threshold by generating signals with random 

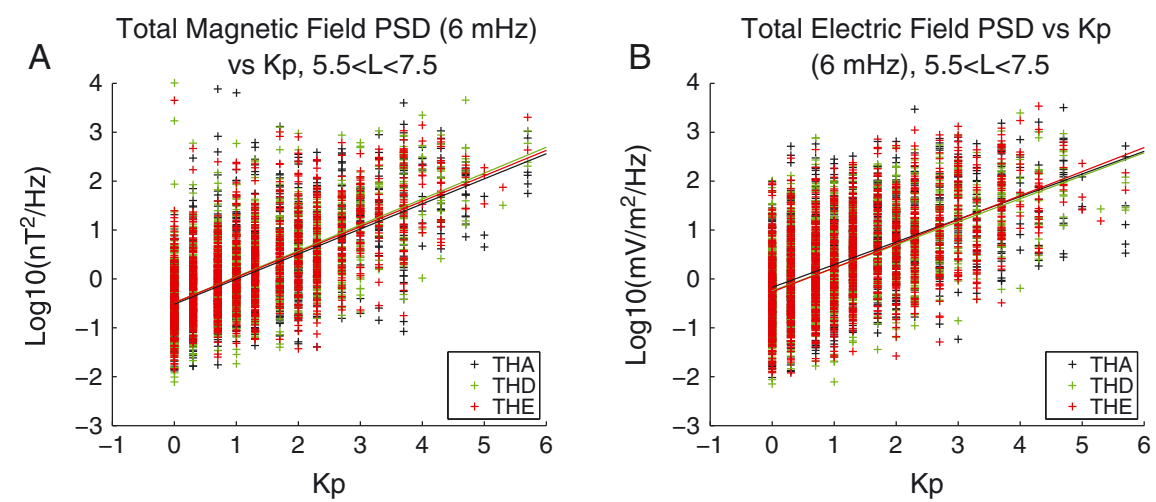

Figure 2. Both electric and magnetic field PSD increase with increasing geomagnetic activity level, and the trend is the same for different THEMIS probes, suggesting there are no sources of systematic error unique to specific probes. (a) The logarithm of the total (sum of three components) magnetic field power spectral density at $6 \mathrm{mHz}$ is plotted versus the $K p$ index. Only data near geosynchronous orbit $(5.5<L<7.5)$ are included, and different colors indicate different THEMIS probes. The PSD measured for each event is shown as a cross, and a line indicates the best fit to $K p$ - the general trend is the same for each probe, and the linear fits are identical (within the uncertainty of the fit). (b) The same as Figure 2a, but for the electric field. The electric field and magnetic field have similar trends, and all probes show the same trends.

fluctuations larger than what we expected for the digitization noise level of each instrument. We found that amplitude detection thresholds of $0.031 \mathrm{nT}$ and $0.043 \mathrm{mV} / \mathrm{m}$ for FGM and EFI, respectively, were high enough to exclude digitization noise. If the RMS amplitude (equation (1)) of perturbations in all components of the electric field or all components of the magnetic field (at any given frequency) were less than these noise levels, we exclude the data from our analysis. These noise levels are frequency independent; since ULF wave power decreases with increasing frequency [e.g., Takahashi and Anderson, 1992], we exclude more high frequency waves from our database than low frequency waves with this restriction. To determine the effect this noise threshold had on our results, we tried increasing the threshold by a factor of roughly 30 (i.e., restricting to large amplitude ULF wave events) - apart from reducing the data available for analysis, this change did not substantially alter our results.

\subsection{Intersatellite Comparisons}

[20] Hartinger et al. [2013] found that the electric field instrument on THA was more strongly affected by conta- mination than on THE. Motivated by this difference, we statistically compared observations from the three THEMIS probes used in this study-THA, THD, and THE-before combining the data together to avoid including systematic biases in our analysis. Takahashi and Anderson [1992] showed that magnetic field perturbations in the inner magnetosphere were strongly correlated with the $K p$ index, or overall geomagnetic activity level. In Figure 2, we show the total (sum of all components) magnetic field and electric field PSD plotted versus $K p$ (we use the $K p$ recorded at the time closest to the PSD measurement). In Figure $2 a$, the magnetic field PSD at $6 \mathrm{mHz}$ is shown on a logarithmic scale versus $K p$ for three probes: THA (black), THD (green), and THE (red). Only data near geosynchronous orbit are shown $(5.5<L<7.5)$. Three lines indicate the best fit to the data for each probe. All three probes see the same trend, to within the error of the fit, of increasing PSD versus $K p$. The same information is shown in Figure $2 b$, but for the electric field. All three probes again see the same trend, and it appears to be the same as for the magnetic field. These data suggest that there are no significant sources of systematic error unique to specific probes. We shall hereafter combine

Table 1. Data Reduction and Total Data Coverage in Days for THA, THD, and THE

\begin{tabular}{|c|c|c|c|}
\hline & THA & THD & THE \\
\hline All data & 1765 & 1765 & 1765 \\
\hline Fast survey & 871 & 886 & 893 \\
\hline In magnetosphere & 715 & 778 & 766 \\
\hline Interval $>60 \mathrm{~min}$ & 627 & 690 & 666 \\
\hline Useable FFT data ( $E / B$ finite, no spikes, edges removed) & 430 & 490 & 477 \\
\hline$\vec{E} \cdot \vec{B}=0$ (spin axis $B$ is large) & 197 & 240 & 238 \\
\hline $\mid$ sun angle $\mid>1.75$ & 169 & 200 & 201 \\
\hline Pc5 above noise ${ }^{b}$ & 124 & 151 & 152 \\
\hline Pc3 above noise ${ }^{b}$ & 44 & 54 & 52 \\
\hline
\end{tabular}

${ }^{a}$ One day is equivalent to 56.25 samples (nonoverlapping FFT windows).

${ }^{\mathrm{b}}$ Indicates number of days of data with Pc5 $(6 \mathrm{mHz})$ or Pc3 $(30 \mathrm{mHz})$ frequency with total electric and magnetic field perturbation amplitudes above the noise floor. 


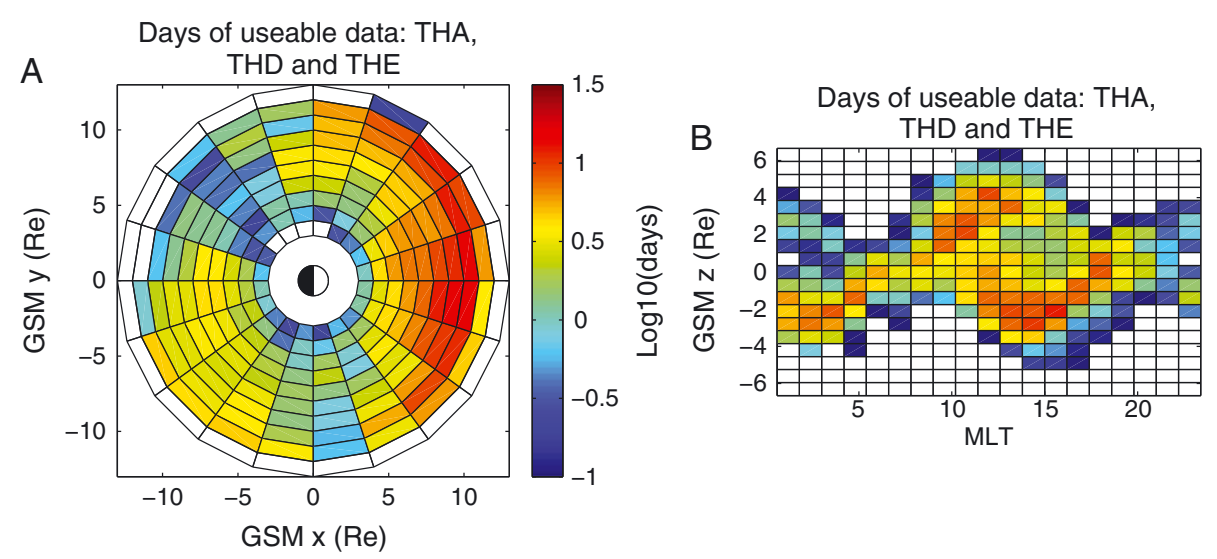

Figure 3. The data coverage from THA, THD, and THE in Log10(days). Only useable (both electric and magnetic field data are available, $E \cdot B=0$ is a valid approximation, |sun angle $\mid$ is below $1.75^{\circ}$ ), unique (THA, THD, and THE are separated by at least 0.5 Re or the observations are at least $25 \mathrm{~min}$ apart) fast survey data are included. One day of data is equivalent to 56.25 samples/FFT windows. (a) Data coverage in the GSM $x y$ plane. Data coverage is not uniform with respect to MLT for a number of reasons, including the choice to alter the times/spatial regions where each probe was in fast survey mode. (b) Data coverage versus GSM $z$ position and MLT. There is a tendency to sample higher GSM $z$ regions on the dayside near noon and near midnight, while the flank regions mostly have data from low GSM $z$ regions.

data from these three probes without concern for introducing systematic errors due to, for example, different probes sampling different regions.

\subsection{Summary-Overview of Database}

[21] Table 1 shows the amount of data, in days, available for each probe after each step in the data reduction process. To avoid double counting ULF wave events when binning the data, if two or more probes make a measurement within 25.6 min (length of FFT window) of each other and when they are separated by less than $0.5 \mathrm{Re}$, we only include data from one probe, with preference given to THE and THD over THA. Figure $3 \mathrm{a}$ shows the GSM $x y$ plane view of data coverage from all probes in $\log _{10}$ (number of days) of useable, nonrepeated data. Although the data sampling is not uniform across all spatial regions, there is ample data in every magnetic local time (MLT) sector and radial distance region for statistical comparisons. Figure $3 \mathrm{~b}$ shows the data coverage versus GSM $z$ position and MLT; there tends to be more samples at high GSM $z$ positions near noon and midnight. This suggests that these regions will tend to include more data from higher magnetic latitudes. Takahashi and Anderson [1992] showed that the magnetic field PSD for ULF waves depends on magnetic latitude, and thus, this irregular sampling may affect our results. We return to this point in later sections.

\section{Results}

[22] In this section, we examine the statistical properties of the ULF wave Poynting vector $(\vec{S}(f))$ at different frequencies.

\subsection{Geosynchronous Orbit}

[23] We first examine the ULF wave Poynting vector in the Pc5 frequency range near geosynchronous orbit. We shall focus on comparisons with Junginger et al. [1985], who examined the Pc5 electric field amplitude and Poynting vector at geosynchronous orbit using the GEOS satellite, due to the large quantity of data (approximately 1 year) they examined. Their use of a different electric field measurement technique is of interest; the electron drift measurement on GEOS was not subject to the same sources of contamination as the double probe measurement used in this study, and thus, systematic differences between these two studies may indicate systematic errors caused by either instrument.

[24] Figure 4 shows the Poynting vector magnitude versus the electric field amplitude for waves with a frequency of $3.9 \mathrm{mHz}$ on a log-log scale. To most closely compare with Junginger et al. [1985], we restrict to regions where the dipole $L$ value is between 5.5 and $7.5 \mathrm{Re}$ (close to geosynchronous orbit) and represent the electric field amplitude using the major axis of the electric field polarization ellipse (calculated using the $x$ and $y$ components of the electric field in FA coordinates [Rankin and Kurtz, 1970]). This figure should be directly compared to in Junginger et al. [1985, Figure 3]; the results are nearly identical. Similar to Junginger et al. [1985], the electric field amplitude varies between 0.022 and $2.8 \mathrm{mV} / \mathrm{m}$ and the Poynting vector magnitude $(|\vec{S}(3.9 \mathrm{mHz})|)$ varies between $3.9 \times 10^{-11} \mathrm{~W} / \mathrm{m}^{2}$ and $1.6 \times 10^{-6} \mathrm{~W} / \mathrm{m}^{2}$. Using their data, Junginger et al. [1985] derived a second power law relationship between the electric field amplitude and Poynting vector magnitude

$$
|\vec{S}|=\frac{\alpha}{\left(2 \mu_{0}\right)}|\vec{E}|^{2}
$$

where $\alpha$ is $1 /\left(1 \times 10^{6} \mathrm{~m} / \mathrm{s}\right)$. This relationship is plotted as a black line on Figure 4 and agrees very well with the data obtained in this study. Thus, there are no apparent sources of systematic error unique to either instrument. Physically, equation (3) implies the preference for a particular 


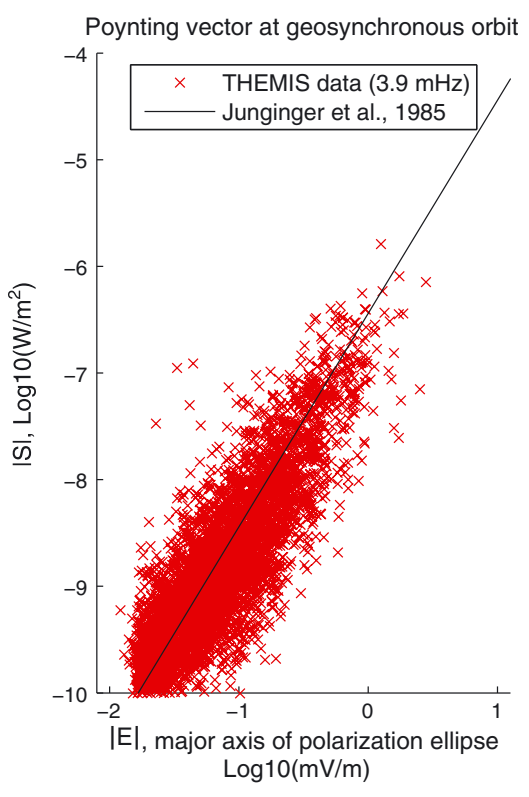

Figure 4. A comparison between THEMIS data and the results of Junginger et al. [1985] at geosynchronous orbit. THEMIS electric and magnetic field data from this study are used to generate the Poynting vector magnitude and electric field amplitude (major axis of polarization ellipse, FA coordinates) at $3.9 \mathrm{mHz}$ in the region near geosynchronous orbit $(5.5<L<7.5)$; these data are plotted as slanted red crosses on a log-log scale. A second power law describing the relationship between the Poynting vector and electric field obtained by Junginger et al. [1985] (using GEOS data at geosynchronous orbit) is plotted as a black line. This figure can be directly compared to in Junginger et al. [1985, Figure 3]. Note that Junginger et al. [1985] used an electron drift instrument rather than a double probe to measure the perturbation electric field, yet the results are still similarthis suggests that neither are substantially affected by unique sources of systematic error.

electromagnetic ULF wave mode in particular magnetic field and plasma conditions; the scatter about this line can be explained by the expected variation in plasma and magnetic field conditions at geosynchronous orbit as well as the possibility that more than one wave mode with a frequency of $3.9 \mathrm{mHz}$ may occur in this region (e.g., both standing Alfvén waves and fast mode waves, which propagate at different speeds).

[25] Junginger et al. [1985] also examined the component of $\vec{S}(3.9 \mathrm{mHz})$ along the background magnetic field and found that during winter, $59 \%$ of vectors were directed toward the northern ionosphere, consistent with expectations for the asymmetry in ionospheric conductivity (higher in southern ionosphere compared to northern ionosphere) [Allan, 1982]. We also restricted our data to summer (20 March to 22 September) and winter (22 September to 20 March) intervals, finding that $53.9 \%$ of Poynting vectors were directed toward the northern ionosphere in winter compared to only $46.7 \%$ in summer, as expected based on the seasonal variation in ionospheric conductivities. These percentages would likely differ more substantially if we restricted to regions in the dayside magnetosphere (as in
Junginger et al. [1985]), low magnetic latitudes, and to standing Alfvén wave events.

\subsection{Spatial Dependence of Poynting Vector: Pc5 Frequencies}

[26] In the next three subsections, we examine the spatial dependence of the Poynting vector at different frequencies, beginning with Pc5 frequencies. We focus specifically on waves with a frequency of $6 \mathrm{mHz}$, which is slightly higher than frequencies examined in previous studies of the Pc5 Poynting vector. Unlike the previous section, in this section we wish to highlight some of the differences between this study and previous studies caused by the examination of different wave frequencies. We shall use GSM coordinates to plot these results. GSM coordinates are well suited for ordering wave activity that is strongly affected by external sources, but they are not as well suited for ordering eigenmodes of the magnetosphere system, which are strongly affected by the terrestrial magnetic field configuration. We tried plotting our results in SM (where the $x y$ plane is the dipole magnetic equator, unlike in GSM) to see how large an effect this had; the effect was minor, and the results were not qualitatively affected. However, we expect that more sophisticated coordinate systems based on empirical magnetic field models would be better suited for studying specific ULF wave modes, and we shall use these in a future study that focuses on ULF wave power variation with distance from the magnetic equator.

[27] Figure 5a shows the GSM $x y$ plane divided into several radial distance and magnetic local time regions, each with a clockdial plot. For each region, the probability density of the Poynting vector at $6 \mathrm{mHz}(\vec{S}(6 \mathrm{mHz}))$ is plotted as a function of vector magnitude and direction (GSM $x y$ projection). In each GSM $x y$ spatial region, we define the probability density as the number of events in a given direction/magnitude bin divided by the total number of events. Note that the probability density is defined separately for each GSM $x y$ spatial region/clockdial plot; the sum of the probability density in each GSM $x y$ spatial region/clockdial plot is one. The scale for each probability density subplot is the same and is shown in the lower left corner; note that the center of these plots is for $1 \times 10^{-12} \mathrm{~W} / \mathrm{m}^{2}$, the outer edge is for $1 \times 10^{-6} \mathrm{~W} / \mathrm{m}^{2}$, and the radial bins are logarithmically spaced to better show the large degree of variation in magnitudes (these probability density plots are explained more thoroughly in the supporting information). Overall, $|\vec{S}(6 \mathrm{mHz})|$ is larger in the outer magnetosphere compared to the inner magnetosphere (compare the outer edge of the clockdial plots at large radial distances to the outer edge of clockdial plots at small radial distances in a given local time sector - the probability density is higher near the outer edge of the clockdial plots at large radial distances, indicating a preference for larger Poynting vector magnitudes), as expected if energy directed earthward from an external driver (e.g, magnetopause perturbations) is progressively lost due to, for example, resonant mode coupling via field line resonance and Joule dissipation in the ionosphere.

[28] A striking feature in Figure 5a is the scatter in the direction of $\vec{S}(6 \mathrm{mHz})$ present in varying amounts at every position (Poynting vector direction is indicated by the angle relative to the positive GSM $x$ axis in each clockdial plot). 

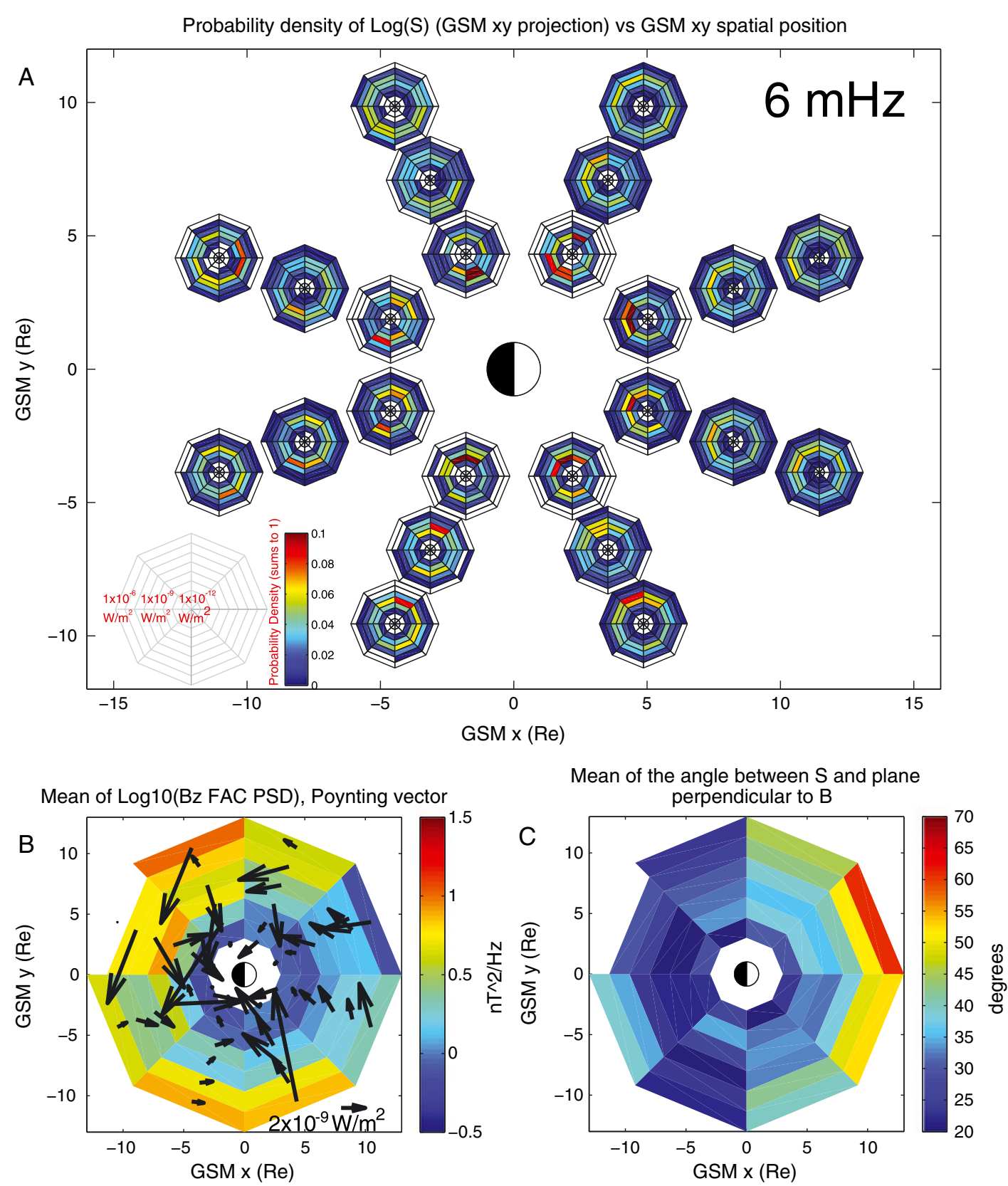

Figure 5. This figure shows the distribution of time-averaged Poynting vector directions and magnitudes for waves with frequencies of $6 \mathrm{mHz}$. (a) A GSM $x y$ view of the Poynting vector probability density (GSM $x y$ projection) in different spatial bins. Each of the small polar plots indicates probability density in color, with Poynting vector magnitudes increasing logarithmically from the center of the plot $\left(1 \times 10^{-12} \mathrm{~W} / \mathrm{m}^{2}\right)$ to the outer edge $\left(1 \times 10^{-6} \mathrm{~W} / \mathrm{m}^{2}\right.$ - see bottom left for scale). Each subplot is in GSM polar coordinates. (b) GSM $x y$ view of the mean parallel magnetic field PSD in color and the mean Poynting vector (GSM $x y$ projection) as black arrows. (c) GSM $x y$ view of the mean of the absolute value of the angle between $\vec{S}$ and the plane perpendicular to $\vec{B}-0$ indicates perpendicular and 90 indicates field-aligned.

For example, in the dayside magnetosphere, there is a preference for earthward directed $\vec{S}(6 \mathrm{mHz})$ at all spatial positions (probability density tends to be higher in that direction at most magnitude levels), as expected if wave energy sources are near or outside the magnetopause. However, in a given spatial bin, a substantial number of $\vec{S}(6 \mathrm{mHz})$ events is directed azimuthally or toward the Sun. This amount of variability was also shown in Chi and Russell [1998] at higher frequencies (Pc3-Pc4); they proposed a bounc- ing wave packet paradigm to explain this scatter. Waves may be excited by an external driver and initially transport energy inward from the magnetopause, but they can be reflected multiple times and eventually propagate/transport energy in a variety of directions. This behavior has been shown in simulations of Pc5 waves: Allan et al. [1987] showed that energy bounced back and forth in the magnetosphere in response to an impulse and Samsonov et al. [2007] simulated the response of the magnetosphere to an 
interplanetary shock, finding that the resulting fast mode shock was reflected in the inner magnetosphere and transported energy back to the magnetopause. Some of this wave energy may leak out of the magnetosphere system through the magnetopause, as suggested in global mode models [Freeman, 2000].

[29] Junginger et al. [1985] and Chi and Russell [1998] examined the mean Poynting vector to identify the nominal direction of energy transfer in different spatial regions. In Figure 5b, we follow this procedure, showing black arrows for the mean $\vec{S}(6 \mathrm{mHz})$ (GSM $x y$ projection) in different spatial bins in the GSM $x y$ plane. Color coding also shows the mean magnetic field PSD for the component along the background magnetic field (FA $z$ component). As in Figure $5 \mathrm{a}$, Figure $5 \mathrm{~b}$ is for wave events with a frequency of $6 \mathrm{mHz}$. In the dayside magnetosphere, the magnetic field PSD is lower than in the nightside magnetosphere and the Poynting vector is directed earthward and duskward, with a stronger earthward preference closer to Earth. These features were previously reported by Chi and Russell [1998], who examined a smaller subset of higher frequency waves (Pc3-Pc4).

[30] A major advantage of this study over previous studies is the large amount of data available, allowing us to more fully describe the Poynting vector at a range of frequencies and spatial locations. As shown in Figure 5a, there is a large amount of scatter in all spatial regions, and this certainly affects the mean values of $\vec{S}(6 \mathrm{mHz})$ shown in Figure 5b. Oppositely directed Poynting vectors of similar magnitude will average to zero, and this has the effect of reducing the magnitude of the mean Poynting vectors as well as obscuring the presence of different ULF wave modes at a given spatial location. Thus, comparisons between Figures $5 \mathrm{a}$ and $5 \mathrm{~b}$ are useful to determine whether mean $\vec{S}(6 \mathrm{mHz})$ is representative of the direction and magnitude of $\vec{S}(6 \mathrm{mHz})$ for the most likely type of wave activity or if multiple types of wave activity with oppositely directed Poynting vectors are present. The duskward preference of Poynting vectors in the dayside magnetosphere is at least partially due to this effect; large magnitude, antisunward and sunward-directed Poynting vectors mostly cancel out, somewhat exaggerating the duskward preference in the mean values.

[31] Junginger [1985] predicted that in the outer flank magnetosphere, the Poynting vector ought to be directed first tailward at large radial distances (close to magnetopause and outside of FLR location), then earthward at intermediate radial distances (closer to FLR), and finally sunward inside of the FLR location. Interestingly, with a few exceptions in the outer dusk magnetosphere, we do not observe this trend. In contrast, Junginger et al. [1985] observed Poynting vectors that were consistent with this trend if the location of their observations was assumed to be earthward of the nominal FLR location, and Sakurai et al. [2001] observed Pc5 Poynting vectors in the outer magnetosphere in a few cases that were directed tailward and toward the Earth (consistent with Junginger [1985]). One possible explanation for this discrepancy is that we are examining a higher frequency. Junginger et al. [1985] reported that the trend was seen most clearly for frequencies below $3 \mathrm{mHz}$ (Figure 4 in that study), and Sakurai et al. [2001] filtered data for frequencies between 2 and $5 \mathrm{mHz}$ (with a peak at $3 \mathrm{mHz}$ ). In contrast, we are examining $6 \mathrm{mHz}$, which may be larger than the typical frequency observed for magnetopause surface waves. We also examined data for the lowest frequency available in our database $(\vec{S}(3 \mathrm{mHz})$ - not shown), and the results were more consistent with Junginger [1985] and Sakurai et al. [2001], with a stronger preference for tailward energy transfer in the outer flank magnetosphere. Thus, the discrepancies between this study and these previous studies can be at least partially attributed to the focus on different frequenciesif our database included frequencies below $3 \mathrm{mHz}$, our results would likely agree more closely with Junginger et al. [1985]. However, consistent with this study, both Junginger et al. [1985] and Sakurai et al. [2001] showed scatter in their Poynting vector observations in the form of some events that were directed in an opposite sense to theoretical expectations for surface waves coupled with FLR.

[32] Figure 5b contains a feature not reported in previous statistical studies of the ULF wave Poynting vector: a preference for sunward directed $\vec{S}(6 \mathrm{mHz})$ in the postmidnight and predawn local time sectors, particularly at larger radial distances - this feature can also be clearly seen in the outermost subplot in the predawn sector in Figure 5a (warmer colors on the sunward side of the subplot indicate a preference for sunward directed $\vec{S}(6 \mathrm{mHz})$; also, several of the bins near the outer edge of the subplot on the sunward side are shaded, indicating a preference for sunward events to have larger $|\vec{S}(6 \mathrm{mHz})|)$. This feature may be due to substormrelated processes, such as bursty bulk flows or drift-bounce resonance with substorm-injected electrons [e.g., Kepko et al., 2001]; the latter mechanism has been indicated as an explanation for sunward propagating ULF waves observed in ground radar data at these local times [James et al., 2013]. It may also be due to the drift mirror instability, which has been shown to drive compressional ULF waves in the dawn sector [Korotova et al., 2009]; however, it is unusual that waves driven by the drift mirror instability would have substantial electric field perturbations, since they are expected to have small propagation speeds (zero in the absence of magnetic field and density gradients) in the plasma rest frame. Whatever the cause, this feature is not clearly seen in the electric or magnetic field PSD and warrants further study. Sunward Poynting vectors are also observed in the dusk sector, consistent with the results of Constantinescu et al. [2009], though their presence is not as clear in the mean values when compared to the dawn sector.

[33] Sakurai et al. [2001] examined the ratio of parallel (to background magnetic field) to perpendicular timeaveraged Pc5 Poynting vectors in the outer flank magnetosphere, finding a strong preference for perpendicular energy transport (the ratio was nearly zero). In a similar analysis, we examine the angle between the Poynting vector and the plane perpendicular to the background magnetic field. Since we are only interested in whether the energy is being transported along the field or perpendicular to the field, we take the absolute value of this angle $-0^{\circ}$ indicates energy transport perpendicular to the background magnetic field, whereas $90^{\circ}$ is for energy transport along the magnetic field. In Figure 5c, we show the mean of the absolute value of the angle, using the same data as in Figures $5 \mathrm{a}$ and $5 \mathrm{~b}$. The angle tends to be smallest on the nightside and in the flank magnetosphere, consistent with Sakurai et al. [2001], who mostly examined data in these regions. In the dayside outer magnetosphere, 
the mean angle becomes much larger; interestingly, the $z$ component of the magnetic field PSD (Figure 5b) is preferentially lower in this region. These observations indicate the presence of standing Alfvén waves in this region for five reasons:

[34] 1. Standing Alfvén waves primarily transport energy along the magnetic field as they gradually damp due to Joule dissipation in the ionosphere.

[35] 2. Six $\mathrm{mHz}$ is consistent with expectations for standing Alfvén wave frequencies in this region [Singer et al., 1981].

[36] 3. Toroidal mode standing Alfvén waves have transverse rather than compressional magnetic perturbations.

[37] 4. Higher angles may be expected on the dayside, where ionospheric conductivities are higher (compared to nightside) and it is easier to set up standing Alfvén waves with large amplitudes via FLR.

[38] 5. Data samples are biased toward higher latitudes in this sector (Figure 3b), where standing Alfvén waves are more likely to be detected relative to fast mode waves (since fast mode waves tend to be confined near the equator) [Lee, 1996].

[39] It is also possible that the large angles on the dayside indicate the presence of a magnetopause surface eigenmode which preferentially transports energy along the field; $6 \mathrm{mHz}$ is higher than frequencies previously reported for surface eigenmodes [Plaschke et al., 2009], but there have been some simulations and observations suggesting that a KelvinHelmholtz unstable dayside magnetopause could cause the growth of surface waves at these frequencies [Takahashi et al., 2012].

[40] We note that lower frequency standing Alfvén waves (or, potentially, magnetopause surface modes) also occur in the dayside outer magnetosphere and that they were observed in the THEMIS data. When examining $3 \mathrm{mHz}$ data in the same manner as the $6 \mathrm{mHz}$ data in Figure $5 \mathrm{c}$, we found qualitatively similar results; in particular, a strong preference for parallel energy transfer in the dayside outer magnetosphere. The largest angles occur in the same GSM location at these different frequencies, consistent with the variability in standing Alfvén wave frequencies (at a fixed position) shown by, for example, Poulter et al. [1984]; these different frequencies overlap here because variations in the plasma mass density, ion composition, and magnetic field were not taken into account when ordering the data.

[41] Item 4 above emphasizes that standing Alfvén waves may be set up via FLR on the dayside more effectively when compared to the nightside. However, there is a competing effect that Joule dissipation rates are larger on the nightside, and this would tend to favor parallel energy transfer on the nightside compared to the dayside (opposite to what is observed). Thus, it is possible that a preference for perpendicular energy transfer on the nightside implies less efficient resonant mode coupling between the fast and shear Alfvén modes on the nightside when compared to the dayside. In this case, fast mode wave energy would be lost more rapidly to, for example, the magnetopause or magnetotail than to mode conversion to shear Alfvén waves, leading to a preference for more perpendicular energy transfer. This is a topic we plan to address in a future study.

\subsection{Spatial Dependence of Poynting Vector: Pc4 Frequencies}

[42] Figure 6 is presented in the same manner as Figure 5 but is for waves with a frequency of $18 \mathrm{mHz}$ rather than $6 \mathrm{mHz}$; note that Figures $6 \mathrm{a}$ and $6 \mathrm{c}$ have the same scales as Figure 5, but the scale in Figure 6b is different than Figure $5 \mathrm{~b}$ to accommodate smaller PSD and Poynting vector values. Figure 6a shows that, as for $\vec{S}(6 \mathrm{mHz})$, there is significant scatter in both the direction and magnitude of $|\vec{S}(18 \mathrm{mHz})|$. However, $18 \mathrm{mHz}$ waves have systematically smaller Poynting vector magnitudes than $6 \mathrm{mHz}$ waves and appear to have a stronger preference to point duskward in the dayside magnetosphere and earthward (positive GSM $x$ direction) in the nightside magnetosphere. Figure 6b shows more clearly the trend for $\vec{S}(18 \mathrm{mHz})$ to point toward dusk in the dayside outer magnetosphere; Chi and Russell [1998] also found this trend for waves with similar frequencies. Compressional waves originating in the ion foreshock could potentially explain this asymmetry, since the ion foreshock is nominally located in the prenoon sector.

[43] Figure $6 \mathrm{~b}$ also shows that in the nightside magnetosphere, the PSD for the magnetic field $z$ component is significantly higher than in other local time sectors (compare with the $6 \mathrm{mHz}$ waves in Figure 5b, where PSD tends to be higher near the flanks); this is consistent with expectations for substorm activity to generate ULF waves in this frequency range [Kepko et al., 2001]. Examination of the mean $\vec{S}(18 \mathrm{mHz})$ reveals that energy associated with this wave activity is primarily directed toward the Earth, consistent with expectations for bursty bulk flows [Angelopoulos et al., 1994; Lessard et al., 1999]. In other regions of the magnetosphere, such as the flanks, the mean Poynting vector exhibits a large degree of variation, even in adjoining spatial bins. This is consistent with the large degree of variation shown in the probability density plots in Figure 6a.

[44] Figure 6c shows somewhat similar trends as Figure $5 \mathrm{c}$; namely, more parallel energy transfer in the dayside magnetosphere. However, when comparing the two figures, the angles tend to be highest in bins at lower radial distances in Figure 6, with the exception of the prenoon sector. This is consistent with our expectations for standing Alfvén waves: lower radial distances generally have higher frequency standing Alfvén waves, and we would thus expect more parallel energy transfer at lower radial distances when examining higher frequency waves.

\subsection{Spatial Dependence of Poynting Vector: Pc3 Frequencies}

[45] Figure 7 shows data for $30 \mathrm{mHz}$ waves and is presented in the same manner as Figures $5(6 \mathrm{mHz})$ and 6 $(18 \mathrm{mHz})$, and the scales in all three panels are identical to Figure 6. Comparing Figures $7 \mathrm{a}$ and $7 \mathrm{~b}$ to the previous two figures, the $|\vec{S}(30 \mathrm{mHz})|$ is systematically lower in all regions. The preference for duskward Poynting vectors in the dayside magnetosphere is similar to that shown in Figure 6 for $18 \mathrm{mHz}$ waves, whereas the trend for earthward directed Poynting vectors for $18 \mathrm{mHz}$ in the nightside magnetosphere is not as strong for $30 \mathrm{mHz}$ waves. The latter feature is consistent with previous findings that substorm related wave activity is peaked at lower frequencies [e.g., Kepko et al., 2001]. 

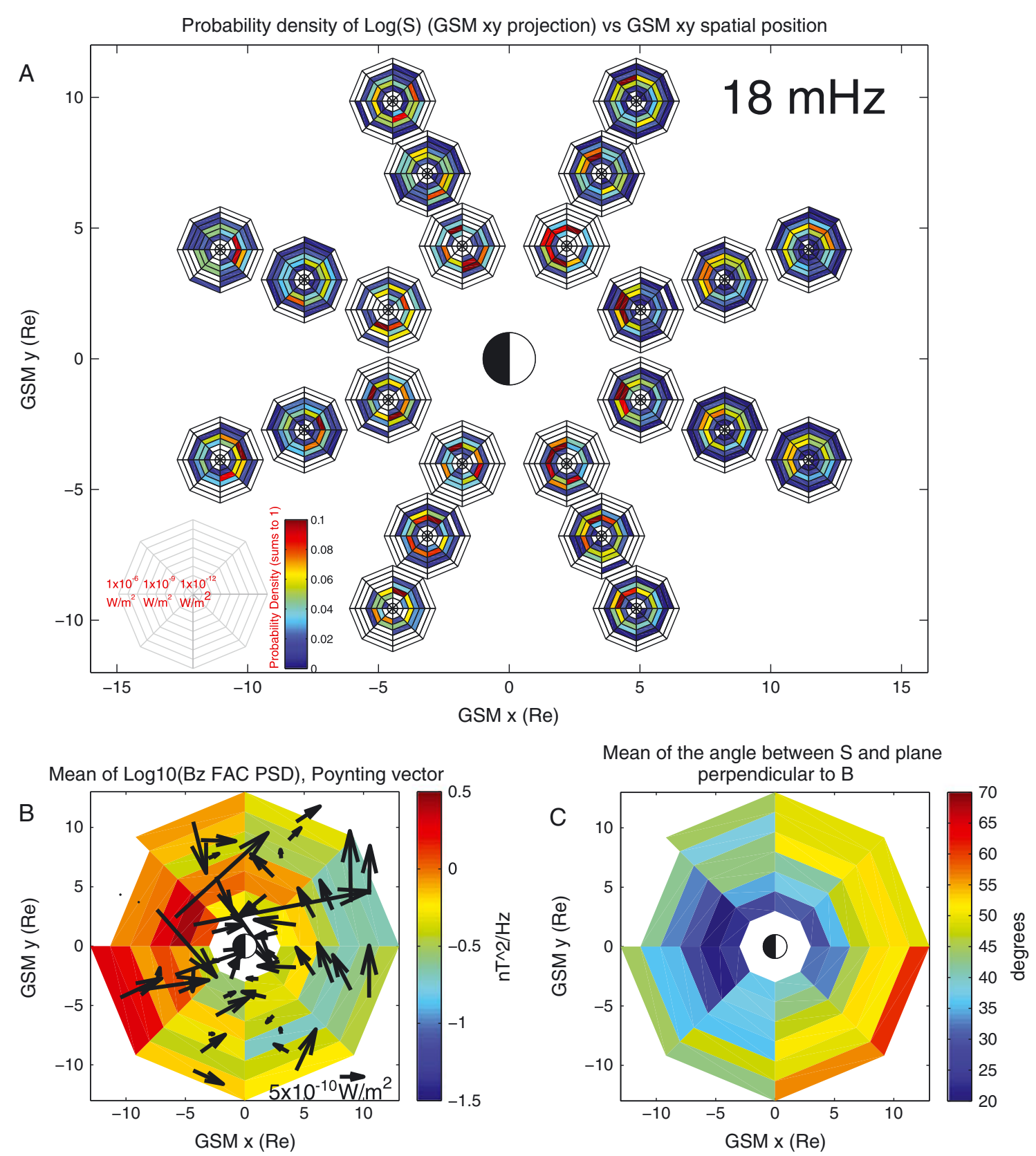

Figure 6. This figure shows the distribution of time-averaged Poynting vector directions and magnitudes for waves with frequencies of $18 \mathrm{mHz}$. (a) A GSM $x y$ view of the Poynting vector probability density (GSM $x y$ projection), in the same format as Figure 5a. (b) GSM $x y$ view of the parallel magnetic field PSD in color and the mean Poynting vector (GSM $x y$ projection) as black arrows. (c) GSM $x y$ view of the mean of the absolute value of the angle between $\vec{S}$ and the plane perpendicular to $\vec{B}-0$ indicates perpendicular and 90 indicates field-aligned.

[46] Figure 7c shows that there is not as strong a local time preference for parallel or perpendicular energy transport when compared to lower frequency waves (Figures 5c and $6 \mathrm{c}$ ): most of the spatial regions have mean angles close to $45^{\circ}$. There is an exception in the outer dusk magnetosphere, where there is a stronger preference for parallel energy transport. This feature is consistent with Lessard et al. [1999], who found a peak occurrence of high frequency $(>10 \mathrm{mHz})$ pulsations with transverse polarization and lower frequency pulsations with radial polarization in the same region; they attributed these two populations to storm time excitation mechanisms associated with the ring current and proposed that the higher frequency activity could potentially be EMIC waves.

\subsection{Frequency Dependence of the Poynting Vector Magnitude/Direction}

[47] As shown in Figures 5, 6, and 7, the Poynting vector magnitude tends to decrease with increasing frequency. Figure 8 shows this trend more clearly, incorporating data 

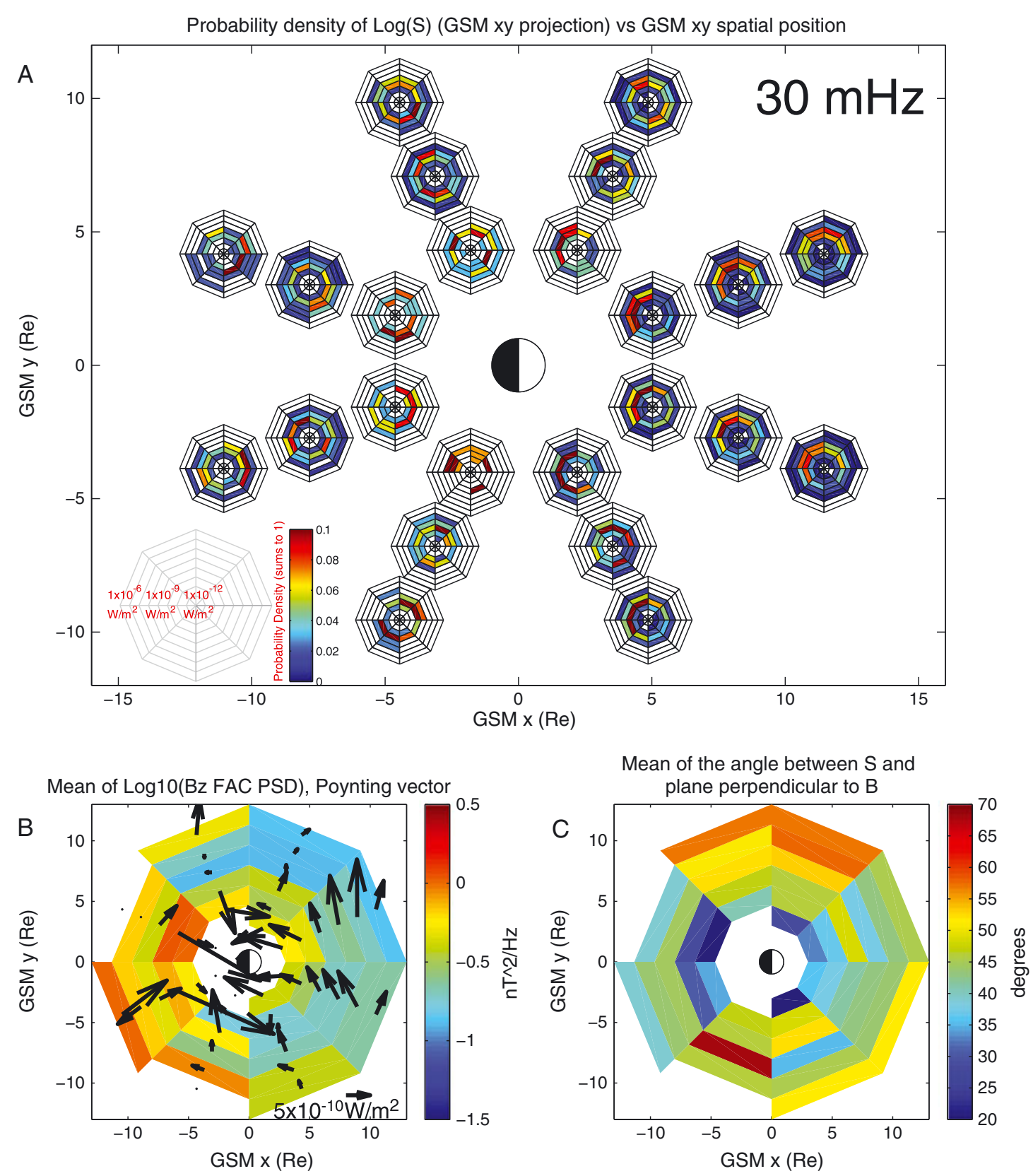

Figure 7. This figure shows the distribution of time-averaged Poynting vector directions and magnitudes for waves with frequencies of $30 \mathrm{mHz}$. (a) A GSM $x y$ view of the Poynting vector probability density (GSM $x y$ projection), in the same format as Figure 5a. (b) GSM $x y$ view of the parallel magnetic field PSD in color and the mean Poynting vector (GSM $x y$ projection) as black arrows. (c) GSM $x y$ view of the mean of the absolute value of the angle between $\vec{S}$ and the plane perpendicular to $\vec{B}-0$ indicates perpendicular and 90 indicates field-aligned.

from different regions and frequencies. The magnitude and direction (relative to background magnetic field) of $\vec{S}$ are shown as a function of frequency for different MLT sectors and radial distances. Figure 8 (top row) is for the dayside magnetosphere and Figure 8 (bottom row) is for the nightside magnetosphere, while Figure 8 (left column) is for radial distances greater than $7 \mathrm{Re}$ and Figure 8 (right column) is for radial distances less than 7 Re. In each panel, the logarithm of the median Poynting vector magnitude (black line) and the median of the absolute value of the angle between $\vec{S}(f)$ and the plane perpendicular to the magnetic field (red line - 0 is for perpendicular propagation and 90 is for parallel) are shown versus frequency. In every region, median $(|\vec{S}(f)|)$ decreases with increasing frequency. This implies that lower frequency waves are the most important for bulk energy transport; this is not that surprising, since both electric and magnetic field PSD also decrease with increasing frequency [e.g., Takahashi and Anderson, 1992] - in other words, more energy is contained in the lower frequency waves, so any bulk transport of energy ought to be larger. However, to our knowledge, this is the first direct measurement of this effect. 


\section{Log of median value of $|S|$, median langle| between $S$ and plane $\perp$ to $B$}
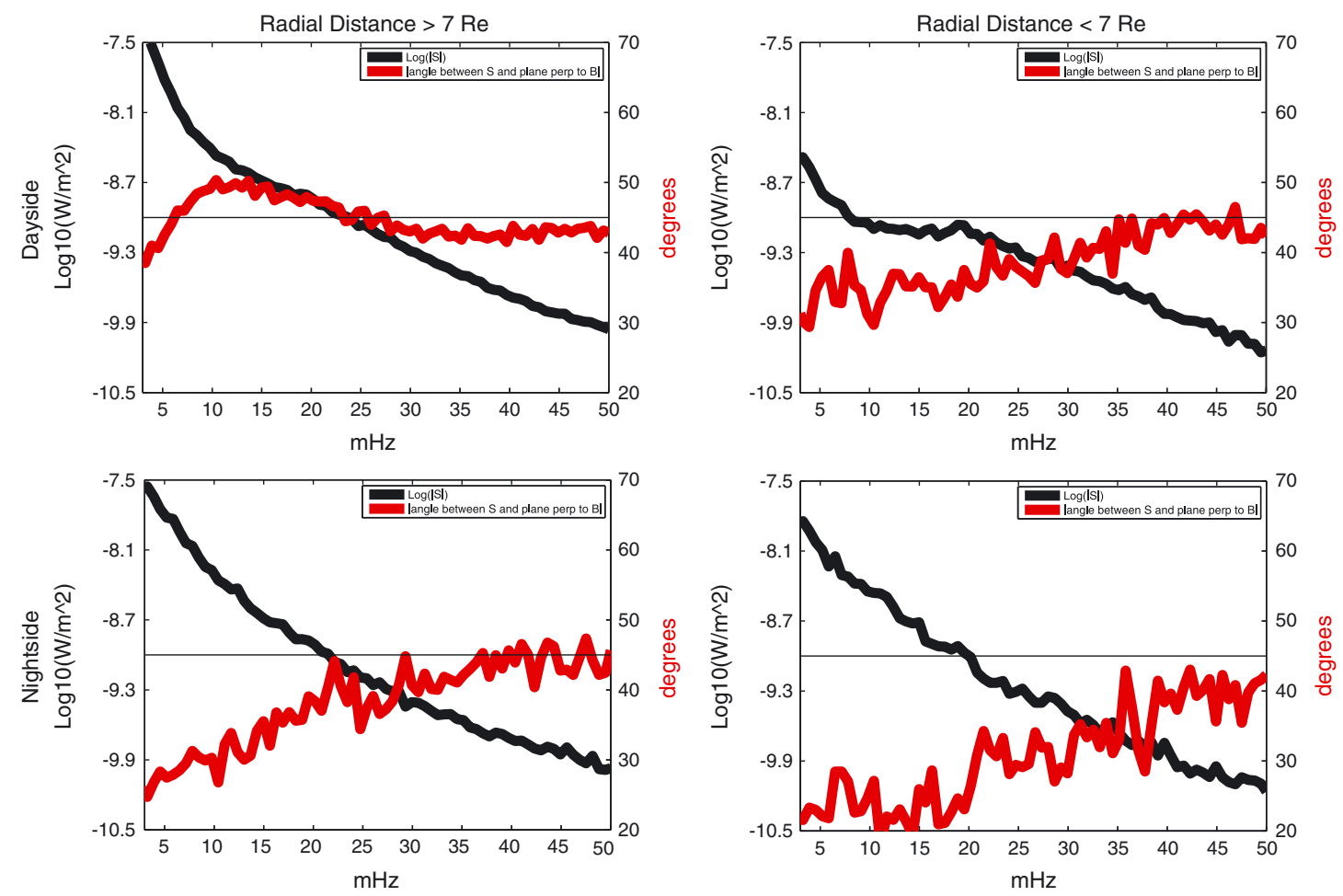

Figure 8. The magnitude and direction (relative to background magnetic field) of the Poynting vector is shown as a function of frequency for different MLT sectors and radial distances. (top row) For the dayside magnetosphere and (bottom row) for the nightside magnetosphere, while (left column) for radial distances greater than $7 \mathrm{Re}$ and (right column) for radial distances less than $7 \mathrm{Re}$. In each panel, the logarithm of the median Poynting vector magnitude (black line) and the median of the absolute value of the angle between the Poynting vector and the plane perpendicular to the magnetic field (red line - 0 is for perpendicular and 90 is for parallel) are shown versus frequency. In every region, the Poynting vector decreases with increasing frequency; there is also a stronger preference for perpendicular energy transport in the nightside magnetosphere and at small radial distances.

[48] There are differences in the Poynting vector magnitude and direction in different regions, as discussed in the context of previous figures. The general shape of the $|\vec{S}(f)|$ spectrum is similar in the dayside and nightside outer magnetosphere (left column); however, the direction of $\vec{S}(f)$ is more likely to be perpendicular to the background magnetic field in the nightside magnetosphere, at least at frequencies less than $30 \mathrm{mHz}$. In the inner magnetosphere (right column), $|\vec{S}(f)|$ is lower than in the outer magnetosphere, both in the dayside and nightside magnetosphere. There is a stronger preference for perpendicular energy transport in the inner magnetosphere compared to the outer magnetosphere at nearly all frequencies, and a stronger preference on the nightside compared to the dayside. We do not expect sampling biases toward high latitudes in certain MLT sectors to have strongly influenced these results (Figure 3b), since both the dayside and nightside sectors include substantial data at large GSM $z$ values $(71 \%$ of observations in the dayside have a distance from the GSM $x y$ plane of greater than $1 \mathrm{Re}$ compared to $73 \%$ on the nightside); if the data were further subdivided into dawn, noon, dusk, and midnight, however, sampling bias would be more important (since the flanks are biased significantly toward lower GSM $z$ values/latitudes).
[49] If Poynting vector directions were randomly distributed - or, in other words, every direction was equally probable-we would expect the median angle relative to the plane perpendicular to the magnetic field to be $30^{\circ}$. Instead, it is typically closer to $45^{\circ}$ (thin black line in each panel), particularly at higher frequencies. This implies a preference for parallel energy transport in nearly all regions of the magnetosphere and at all frequencies. This is somewhat surprising, since these observations were made close to the magnetic equator, where perpendicular energy transport associated with fast mode waves should be strongest (since they tend to be confined near the equator) and parallel energy transport associated with standing Alfvén waves should be weaker [Allan, 1982; Takahashi and Anderson, 1992; Lee, 1996]. These results suggest that Joule dissipation in the ionosphere and the field-aligned acceleration of auroral electrons are the most important sinks of ULF wave energy in the magnetosphere (compared to, for example, the magnetopause or magnetotail). To our knowledge, there have been no attempts to make a quantitative prediction for the relative importance of parallel versus perpendicular energy transport averaged over all conditions. Usually, models focus on specific ULF wave modes and use various assumptions for ionospheric conductivity, radial density 
profiles, and idealized driving conditions. These results can thus provide guidelines for future models attempting to study ULF wave energy transport during nominal conditions in the magnetosphere. We also expect that by binning our results further by magnetic latitude, season, and specific driving conditions, direct comparisons could be made with existing models that calculate the ULF wave Poynting vector. For example, Lysak and Lee [1992] showed different ULF responses to monochromatic and impulsive driving conditions, and Allan et al. [1987] showed that initial, transient responses to an impulsive driver can generate significant energy flow (differing from the time asymptotic behavior expected in idealized ULF wave models). This suggests that by separating impulsive driving events from monochromatic driving events and by separating initial, transient responses from later responses, our results would agree more closely with ULF wave models with idealized driving conditions.

[50] The shape of the $|\vec{S}(f)|$ spectrum in the dayside inner magnetosphere is unique. As shown in Figure 8 (top right), the spectrum flattens between roughly 8 and $20 \mathrm{mHz}$ (black line); this same feature also appears in the total magnetic field PSD spectrum (not shown). This could indicate the presence of a resonance, either at the plasmapause or in the plasmasphere, or could simply be a result of the fact that higher frequency fast mode waves are not attenuated as strongly as lower frequency waves as they propagate from the outer to the inner magnetosphere [Lee, 1996; Takahashi et al., 2010]. We do not think this feature indicates the presence of a field line resonance; if that were the case, we would have expected the median angle between the Poynting vector and the plane perpendicular to the magnetic field to increase in this frequency range to be consistent with the behavior of large amplitude standing Alfvén waves; however, it remains steady (red line).

\section{Summary}

[51] We have presented a survey of ULF wave Poynting vector observations covering the frequency range from 3 to $50 \mathrm{mHz}$ and the spatial region from 3 to $13 \mathrm{Re}$ near the magnetic equatorial plane. We find that the magnitude of the Poynting vector decreases with increasing frequency/decreasing radial distance and that energy transfer is anisotropic, with energy preferentially transferred along the background magnetic field. We suggest that the preference for parallel energy transfer, coupled with the fact that these observations are near the magnetic equatorial plane (where there ought to be the strongest preference for perpendicular energy transfer), suggests that Joule dissipation in the ionosphere and the acceleration of auroral electrons are the largest sinks of ULF wave energy in the magnetosphere. We examined the Poynting vector magnitude and direction at different frequencies and in different regions, finding several key features:

[52] 1. Preference for duskward and earthward energy transfer on the dayside at all frequencies.

[53] 2. Preference for sunward Poynting vectors in the predawn sector at Pc5 frequencies potentially associated with substorm processes (bursty bulk flows, drift-bounce resonance with injected electrons).
[54] 3. Preference for parallel Poynting vectors likely associated with standing Alfvén waves near the dayside magnetopause at Pc5 and Pc4 frequencies.

[55] 4. Strong earthward energy transfer in the nightside magnetosphere for $\mathrm{Pi} 2 / \mathrm{Pc} 4$ frequencies likely caused by substorm processes (e.g., bursty bulk flows).

[56] 5. Preference for parallel Poynting vectors in the dusk local time sector at Pc3 frequencies.

[57] The Poynting vector measurement was essential for identifying several of these features, as they could not be seen or inferred from investigation of the electric and magnetic field PSD observations.

[58] Poynting vector magnitudes ranged from roughly $1 \times 10^{-11}$ to $1 \times 10^{-5} \mathrm{~W} / \mathrm{m}^{2}$. At the upper end of this range, bulk energy transport is comparable to other major energy transport mechanisms in the Earth's magnetosphere. For example, a damped, standing Alfvén wave energy transfer rate of roughly $1 \times 10^{-7} \mathrm{~W} / \mathrm{m}^{2}$ in the magnetic equatorial plane maps to a transfer rate of roughly $1 \times 10^{-3} \mathrm{~W} / \mathrm{m}^{2}$ in the topside ionosphere [Hartinger et al., 2011]. Similar numbers, when integrated over the expected lifetime of the Alfvén wave and the spatial extent of the wave, are comparable to the energy released during a small substorm and can substantially heat the ionosphere [Greenwald and Walker, 1980; Rae et al., 2007]. These energy transfer rates are larger than higher frequency electromagnetic plasma waves sampled in similar regions, such as whistler mode chorus [e.g., Santolik et al., 2010; Li et al., 2013] (note that the Poynting vector observations in these studies have units of energy flux per Hz, whereas in this study, they are in units of energy flux); this is expected, since higher frequency waves tend to have much smaller wave amplitudes/PSD compared to ULF waves and should thus be less important for net electromagnetic energy transport (although they play very important roles in energy transport via wave-particle interactions).

[59] We found that although there were preferred directions for the Poynting vector in different regions and at different frequencies, there was a substantial amount of scatter. We showed that this scatter affects mean values of the Poynting vector in a given spatial region, and it can lead to substantially underestimated Poynting vector magnitudes in that region (since oppositely directed Poynting vectors cancel out). Physically, it also means that there are not strongly preferred directions for net wave energy transport in most regions of the magnetosphere, perhaps due to externally driven wave activity being reflected multiple times in the magnetosphere or the presence of multiple wave modes [e.g., Allan et al., 1987; Chi and Russell, 1998; Samsonov et al., 2007]. We also found evidence for several wave modes that, while clear in the Poynting vector observations, were not clear in examination of wave PSD; in particular, higher frequency ( $\mathrm{Pc} 3$ ) parallel propagating waves in the dusk sector (possibly related to wave activity reported by Lessard et al. [1999]) and sunward propagating Pc5 waves in the postmidnight/predawn sector (possibly related to wave activity reported by James et al. [2013]).

[60] The Poynting vector is a useful diagnostic for ULF wave mode identification and an important tool for determining the relative importance of ULF waves compared to other magnetospheric energy transfer mechanisms. In a future study, we will use this database to study energy transfer along the background magnetic field and its impact on 
the ionosphere. We will also examine the effect of ULF wave propagation directions on the radial transport of radiation belt electrons.

[61] Acknowledgments. M.D. Hartinger was funded through NSF grant AGS-1230398. K. Takahashi was funded by NASA grant NNX13AE02G. We acknowledge NASA THEMIS contract NAS5-02099 and thank C.W. Carlson and J.P. McFadden for the use of the ESA data, D. Larson and R.P. Lin for the use of SST data, J.W. Bonnell and F.S. Mozer for use of EFI data, and K.H. Glassmeier, U. Auster, and W. Baumjohann for the use of FGM data provided under the lead of the Technical University of Braunschweig and with financial support through the German Ministry for Economy and Technology and the German Center for Aviation and Space (DLR) under contract 50 OC 0302. We thank the NASA Space Science Data facility for use of solar wind data and geomagnetic activity indices.

[62] Robert Lysak thanks William Allan and an anonymous reviewer for their assistance in evaluating this paper.

\section{References}

Allan, W. (1982), Phase variation of ULF pulsations along the geomagnetic field-line, Planet. Space Sci., 30, 339-346, doi:10.1016/00320633(82)90039-3.

Allan, W., E. M. Poulter, and S. P. White (1987), Hydromagnetic wave coupling in the magnetosphere-magnetic fields and Poynting fluxes, Planet. Space Sci., 35, 1181-1192, doi:10.1016/0032-0633(87)90024-9.

Anderson, B. J., M. J. Engebretson, S. P. Rounds, L. J. Zannetti, and T. A. Potemra (1990), A statistical study of Pc3-Pc5 pulsations observed by the AMPTE/CCE Magnetic Fields Experiment, 1. Occurrence distributions, J. Geophys. Res., 95, 10,495-10,523, doi:10.1029/JA095iA07p10495.

Angelopoulos, V., C. F. Kennel, F. V. Coroniti, R. Pellat, M. G. Kivelson, R. J. Walker, C. T. Russell, W. Baumjohann, W. C. Feldman, and J. T. Gosling (1994), Statistical characteristics of bursty bulk flow events, $J$. Geophys. Res., 99, 21,257-21,280, doi:10.1029/94JA01263.

Auster, H. U., et al. (2008), The THEMIS fluxgate magnetometer, Space Sci. Rev., 141, 235-264, doi:10.1007/s11214-008-9365-9.

Bonnell, J. W., F. S. Mozer, G. T. Delory, A. J. Hull, R. E. Ergun, C. M. Cully, V. Angelopoulos, and P. R. Harvey (2008), The electric field instrument (EFI) for THEMIS, Space Sci. Rev., 141, 303-341, doi:10.1007/s11214-008-9469-2.

Chi, P. J., and C. T. Russell (1998), Phase skipping and Poynting flux of continuous pulsations, J. Geophys. Res., 103, 29,479-29,491, doi:10.1029/98JA02101.

Constantinescu, O. D., et al. (2009), THEMIS observations of duskside compressional Pc5 waves, J. Geophys. Res., 114, A00C25, doi:10.1029/2008JA013519.

Elkington, S. R., M. K. Hudson, and A. A. Chan (2003), Resonant acceleration and diffusion of outer zone electrons in an asymmetric geomagnetic field, J. Geophys. Res., 108(A3), 1116, doi:10.1029/2001JA009202.

Freeman, M. P. (2000), Effect of magnetopause leakage on the lifetime of magnetospheric cavity modes, J. Geophys. Res., 105, 5463-5470, doi:10.1029/1999JA900483.

Frey, S., V. Angelopoulos, M. Bester, J. Bonnell, T. Phan, and D. Rummel (2008), Orbit design for the THEMIS mission, Space Sci. Rev., 141, 61-89, doi:10.1007/s11214-008-9441-1.

Greenwald, R. A., and A. D. M. Walker (1980), Energetics of long period resonant hydromagnetic waves, Geophys. Res. Lett., 7, 745-748, doi:10.1029/GL007i010p00745.

Hartinger, M., V. Angelopoulos, M. B. Moldwin, K.-H. Glassmeier, and Y. Nishimura (2011), Global energy transfer during a magnetospheric field line resonance, Geophys. Res. Lett., 38, L12101, doi:10.1029/2011GL047846.

Hartinger, M., V. Angelopoulos, M. B. Moldwin, Y. Nishimura, D. L. Turner, K.-H. Glassmeier, M. G. Kivelson, J. Matzka, and C. Stolle (2012), Observations of a Pc5 global (cavity/waveguide) mode outside the plasmasphere by THEMIS, J. Geophys. Res., 117, A06202, doi:10.1029/2011JA017266.

Hartinger, M. D., V. Angelopoulos, M. B. Moldwin, K. Takahashi, and L. B. N. Clausen (2013), Statistical study of global modes outside the plasmasphere, J. Geophys. Res. Space Physics, 118, 804-822, doi:10.1002/jgra.50140.

Jacobs, J. A., Y. Kato, S. Matsushita, and V. A. Troitskaya (1964), Classification of geomagnetic micropulsations, J. Geophys. Res., 69, 180-181, doi:10.1029/JZ069i001p00180.

James, M. K., T. K. Yeoman, P. N. Mager, and D. Y. Klimushkin (2013), The spatio-temporal characteristics of ULF waves driven by substorm injected particles, J. Geophys. Res. Space Physics, 118, 1737-1749, doi:10.1002/jgra.50131.
Junginger, H. (1985), Poynting vector as a diagnostic of hydromagnetic wave structure, J. Geophys. Res., 90, 4155-4163, doi:10.1029/JA090iA05p04155.

Junginger, H., G. Haerendel, and F. Melzner (1985), A statistical study of wave Poynting vectors measured during long-period magnetospheric pulsations at geostationary orbit, J. Geophys. Res., 90, 8301-8307, doi:10.1029/JA090iA09p08301.

Keiling, A., J. R. Wygant, C. Cattell, W. Peria, G. Parks, M. Temerin, F. S. Mozer, C. T. Russell, and C. A. Kletzing (2002), Correlation of Alfvén wave Poynting flux in the plasma sheet at 4-7 $R_{E}$ with ionospheric electron energy flux, J. Geophys. Res., 107(A7), 1132, doi:10.1029/2001JA900140.

Keiling, A., J. R. Wygant, C. A. Cattell, F. S. Mozer, and C. T. Russell (2003), The global morphology of wave Poynting flux: Powering the aurora, Science, 299, 383-396, doi:10.1126/science. 1080073.

Kepko, L., M. G. Kivelson, and K. Yumoto (2001), Flow bursts, braking, and Pi2 pulsations, J. Geophys. Res., 106, 1903-1916, doi:10.1029/2000JA000158.

Korotova, G. I., D. G. Sibeck, V. Kondratovich, V. Angelopoulos, and O. D. Constantinescu (2009), THEMIS observations of compressional pulsations in the dawn-side magnetosphere: A case study, Ann. Geophys., 27, 3725-3735, doi:10.5194/angeo-27-37252009.

Lee, D.-H. (1996), Dynamics of MHD wave propagation in the low-latitude magnetosphere, J. Geophys. Res., 101, 15,371-15,386, doi:10.1029/96JA00608

Lessard, M. R., M. K. Hudson, and H. Lühr (1999), A statistical study of Pc3-Pc5 magnetic pulsations observed by the AMPTE/Ion Release Module satellite, J. Geophys. Res., 104, 4523-4538, doi:10.1029/1998JA900116.

Li, W., J. Bortnik, R. M. Thorne, C. M. Cully, L. Chen, V. Angelopoulos, Y. Nishimura, J. B. Tao, J. W. Bonnell, and O. LeContel (2013), Characteristics of the Poynting flux and wave normal vectors of whistler-mode waves observed on THEMIS, J. Geophys. Res. Space Physics, 118, 1461-1471, doi:10.1002/ jgra.50176.

Loto'aniu, T. M., B. J. Fraser, and C. L. Waters (2005), Propagation of electromagnetic ion cyclotron wave energy in the magnetosphere, $J$. Geophys. Res., 110, A07214, doi:10.1029/2004JA010816.

Lysak, R. L., and D. H. Lee (1992), Response of the dipole magnetosphere to pressure pulses, Geophys. Res. Lett., 19, 937-940, doi:10.1029/92GL00625.

Lysak, R. L., and Y. Song (2003), Kinetic theory of the Alfvén wave acceleration of auroral electrons, J. Geophys. Res., 108(A4), 8005, doi:10.1029/2002JA009406.

McFadden, J. P., C. W. Carlson, D. Larson, M. Ludlam, R. Abiad, B. Elliott, P. Turin, M. Marckwordt, and V. Angelopoulos (2008), The THEMIS ESA plasma instrument and in-flight calibration, Space Sci. Rev., 141, 277-302, doi:10.1007/s11214-008-9440-2.

Plaschke, F, K.-H. Glassmeier, H. U. Auster, O. D Constantinescu, W. Magnes, V. Angelopoulos, D. G. Sibeck, and J. P. McFadden (2009), Standing Alfvén waves at the magnetopause, Geophys. Res. Lett., 36, L02104, doi:10.1029/2008GL036411.

Poulter, E. M., W. Allan, J. G. Keys, and E. Nielsen (1984), Plasmatrough ion mass densities determined from ULF pulsation eigenperiods, Planet. Space Sci., 32, 1069-1078, doi:10.1016/0032-0633 (84)90132-6.

Rae, I. J., C. E. J. Watt, F. R. Fenrich, I. R. Mann, L. G. Ozeke, and A. Kale (2007), Energy deposition in the ionosphere through a global field line resonance, Ann. Geophys., 25, 2529-2539, doi:10.5194/angeo-25-25292007.

Rae, I. J., C. E. J. Watt, K. R. Murphy, H. U. Frey, L. G. Ozeke, D. K. Milling, and I. R. Mann (2012), The correlation of ULF waves and auroral intensity before, during and after substorm expansion phase onset, $J$ Geophys. Res., 117, A08213, doi:10.1029/2012JA017534.

Rankin, D., and R. Kurtz (1970), Statistical study of micropulsation polarizations, J. Geophys. Res., 75, 5444-5458, doi:10.1029/ JA075i028p05444.

Sakurai, T., Y. Tonegawa, Y. Shinkai, K. Yumoto, S. Kokubun, K. Tsuruda, and T. Mukai (2001), Poynting vectors of Pc5 pulsations observed by the GEOTAIL satellite in the dayside outer magnetosphere, Earth Planets Space, 53, 843-849.

Samsonov, A. A., D. G. Sibeck, and J. Imber (2007), MHD simulation for the interaction of an interplanetary shock with the Earth's magnetosphere, J. Geophys. Res., 112, A12220, doi:10.1029/2007JA012627.

Santolík, O., J. S. Pickett, D. A. Gurnett, J. D. Menietti, B. T. Tsurutani, and O. Verkhoglyadova (2010), Survey of Poynting flux of whistler mode chorus in the outer zone, J. Geophys. Res., 115, A00F13, doi:10.1029/2009JA014925. 
Sibeck, D. G., and V. Angelopoulos (2008), THEMIS science objectives and mission phases, Space Sci. Rev., 141, 35-59, doi:10.1007/s11214008-9393-5.

Singer, H. J., D.-J. Southwood, R. J. Walker, and M. G. Kivelson (1981), Alfvén wave resonances in a realistic magnetospheric magnetic field geometry, J. Geophys. Res., 86, 4589-4596, doi:10.1029/ JA086iA06p04589.

Takahashi, K., and B. J. Anderson (1992), Distribution of ULF energy $(f<80 \mathrm{mHz})$ in the inner magnetosphere: A statistical analysis of AMPTE CCE magnetic field data, J. Geophys. Res., 97, 10,751-10,773, doi:10.1029/92JA00328.

Takahashi, K., D.-H. Lee, M. Nosé, R. R. Anderson, and W. J. Hughes (2003), CRRES electric field study of the radial mode structure of Pi2 pulsations, J. Geophys. Res., 108(A5), 1210, doi:10.1029/ 2002JA009761.
Takahashi, K., et al. (2010), Multipoint observation of fast mode waves trapped in the dayside plasmasphere, J. Geophys. Res., 115, A12247, doi:10.1029/2010JA015956.

Takahashi, K., K. Yumoto, S. G. Claudepierre, E. R. Sanchez, O. A Troshichev, and A. S. Janzhura (2012), Dependence of the amplitude of Pc5-band magnetic field variations on the solar wind and solar activity, J. Geophys. Res., 117, A04207, doi:10.1029/ 2011JA017120.

Tamao, T. (1965), Transmission and coupling resonance of hydromagnetic disturbances in the non-uniform Earth's magnetosphere, Sci. Rep. Tohoku Univ., Ser. 5, Geophys., 17(2), 43-72.

Turner, D. L., Y. Shprits, M. Hartinger, and V. Angelopoulos (2012), Explaining sudden losses of outer radiation belt electrons during geomagnetic storms, Nat. Phys., 8, 208-212, doi:10.1038/ nphys 2185 . 